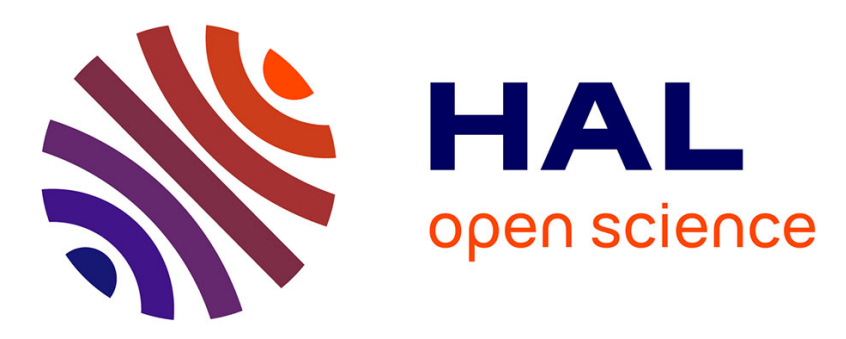

\title{
Template-Based Tractography for Clinical Neonatal Diffusion Imaging Data
}

\author{
Natasha Lepore, Fernando Yepes, Yi Lao, Ashok Panigraphy, Rafael \\ Cheschin, Subhashree Ravichandran, Marvin D. Nelson, Pierre Fillard
}

\section{To cite this version:}

Natasha Lepore, Fernando Yepes, Yi Lao, Ashok Panigraphy, Rafael Cheschin, et al.. Template-Based Tractography for Clinical Neonatal Diffusion Imaging Data. Template-Based Tractography for Clinical Neonatal Diffusion Imaging Data, SPIE, Feb 2012, San Francisco CA, United States. hal-00675337

\section{HAL Id: hal-00675337 https://hal.inria.fr/hal-00675337}

Submitted on 4 Mar 2012

HAL is a multi-disciplinary open access archive for the deposit and dissemination of scientific research documents, whether they are published or not. The documents may come from teaching and research institutions in France or abroad, or from public or private research centers.
L'archive ouverte pluridisciplinaire HAL, est destinée au dépôt et à la diffusion de documents scientifiques de niveau recherche, publiés ou non, émanant des établissements d'enseignement et de recherche français ou étrangers, des laboratoires publics ou privés. 


\title{
Template-Based Tractography for Clinical Neonatal Diffusion Imaging Data
}

\author{
Natasha Leporé ${ }^{a, c, *}$ and Fernando Yepes ${ }^{b, c, *}, \mathrm{Yi} \mathrm{Lao}^{c}$, Ashok Panigrahy $^{c, e}$, Rafael Ceschin ${ }^{e}$, \\ Subhashree Ravichandran ${ }^{c}$, Marvin D. Nelson $^{c}$, Pierre Fillard $^{d}$ \\ ${ }^{a}$ University of Southern California, 900 W 34th St, Los Angeles-CA, USA; \\ ${ }^{b}$ Instituto de Investigaciones Biomédicas de Barcelona, Rosselló, 161, Barcelona, Spain; \\ ${ }^{c}$ Children's Hospital Los Angeles, 4650 Sunset Blvd, Los Angeles-CA, USA; \\ ${ }^{d}$ Parietal Research Team, INRIA Parc Orsay Universit 4, rue Jacques Monod, Paris, France; \\ ${ }^{e}$ Children's Hospital of Pittsburgh, 4401 Penn Avenue Pittsburgh, Pittsburgh-PA, USA
}

\begin{abstract}
In imaging studies of neonates, particularly in the clinical setting, diffusion tensor imaging-based tractography is typically unreliable due to the use of fast acquisition protocols that yield low resolution and signal-to-noise ratio (SNR). These image acquisition protocols are implemented with the aim of reducing displacement artifacts that may be produced by the movement of the neonate's head during the scanning session. ${ }^{1}$ In addition, axons are not yet fully myelinated in neonates. ${ }^{1-3}$ As a result, the water molecule's movements are not as constrained as in older brains, making it even more difficult to define structure by means of diffusion profiles. Here, we introduce a post-processing method that overcomes some of the difficulties described above, allowing the determination of reliable tracts in newborns. We test our method using neonatal data and in particular, we successfully extract a limbic and some association and commisural fibers, all of which are typically difficult to obtain by direct tractography. The method is further validated through visual inspection by expert pediatric neuroradiologists.
\end{abstract}

Keywords: DTI, Neonates, template, tractography, CNR, SNR, tensor registration, tensor rotation, warped fibers, fiber tracking.

\section{INTRODUCTION}

Diffusion tensor imaging (DTI) has become the most used tool for in-vivo brain auscultation. This technique is capable of creating contrast between White Matter (WM) and Grey Matter (GM) based on the water diffusion profiles. ${ }^{4,5}$ From a simplistic approach, DTI measures how hindered is the random displacement of water molecules. The water diffusion lecture is a macro-manifestation of an existing underlying micro-structure. This is why, zones with highly isotropic diffusion profiles are associated to highly ordered and dense structures, proper of WM. In contrast, low isotropic diffusion indexes are associated to low order and less density of structures, thus GM. ${ }^{6}$

Besides the underlying structure, there are other physiological and technical aspects that affect the DTI readings. Among the physiological aspects we have: Axon packaging, cellular permeability to water, tissue water content and presence of myeline. ${ }^{1}$ In the technical side, DTI measurements may vary primarily with the spatial resolution used, the diffusion elapsed time -related to bvalue- and how well we analyze the possible paths of water displacement in the three dimensional space -namely number of diffusion gradient directions-.

Scanning neonates is challenging in both mentioned aspects. Physiologically, due to low content of myeline, the similar content of water in WM and GM, and the low axonal packaging in the overall developing brain. Technically, due to the difficulties associated to keeping a non-sedated neonate static into the scanner the desired time to accomplishing a good SNR, a good spatial resolution and a relatively high angular resolution. This is why, clinical DTI scanning procedures in neonates often use very rapid sequences at expense of image quality.

The physiological facts posted here as DTI complications, are not static. Since the end of the first trimester of pregnancy, brain structures start developing and this development has been documented until the age of 5-6

\footnotetext{
* Equal contribution.
} 
years old. ${ }^{2}$ This maturity process includes increments in: myeline content, the differentiation in the content of water between GM and WM structures and amount of axon packaging. All these manifestations of development are detectable trough DTI. From here the importance of this technique in understanding the brain maturity process.

As mentioned above, DTI methods estimate the water displacement. This happens mostly along axons, thus this technique can also elucidate the pathways of the neuronal trends. This is know as tractography and applied to the brain studies, presents robust insights about brain connectivity. In terms of brain maturity, the connectivity between brain zones is more meaningful than the contrast between brain structures itself. ${ }^{7,8}$ In particular, it is known that some limbic tracts such as the fornix are established after 19-20 gestational weeks, ${ }^{2}$ so it is supposed to be visible under DTI methods, nevertheless, it is hardly watchable in the clinical datasets. Some association fibers such as the Inferior-Fronto-Oscipital-Fascilus (IFOF) and the Inferior-Longitudinal-Fascilus (IFL) are not completely developed in term babies. ${ }^{2}, 9$ Commisural fibers such as the Posterior-Thalamic-Rad (PTR) and the Anterior-thalamic-Rad (ATR) happens to develop mostly between the last trimester of pregnancy and, according to Prayer et al, ${ }^{10}$ these tracts are not fully myelinated until gestional week $41^{\text {th }}$. All the mentioned neuronal structures become markers of the brain maturity process starting in the fetus when the brain begins its formation and having a critical development during first years of life. ${ }^{11}$

We have created a post processing pipeline that overcomes the limitations normally encountered in DTI neonatal studies, by making the images become resourceful. Our procedure creates a gold standard volume with high quality specifications where the connective paths are easily obtained. We tested the efficacy of our procedure by extracting some of the mentioned development marker tracts, including: The fornix, the IFOF, the ILF, the PTR and the ATR. The procedure was tested in retrospective clinical data of newborns, which has a low image quality due to the fast acquisition sequence used and moreover, where the marker tracts could not be extracted by direct tractography.

\section{MATERIALS AND METHODS}

\subsection{Neonates-DTI used experiments}

Six brain DTI scans of term neonates (post-conception ages 46.8 weeks \pm 3.18 ) with normal MRI scans were recovered from the database of the Children's Hospital of Los Angeles (CHLA). All images were acquired with a $1.5 \mathrm{~T}$ GE scanner using 25 gradient directions, a neonatal head coil, b-value $=700 \mathrm{~s} / \mathrm{mm} 2$ and echo planar imaging (EPI) sequence. Slice thickness is $5 \mathrm{~mm}$. Due to the large available database, selecting acquisitions with similar spatial dimensions and even similar original clinical objectives was straightforward. Nevertheless, our procedure includes a re-sampling step so that images with different spatial resolutions may also be used. The use of this retrospective data was approved by the local IRB at the CHLA.

\subsection{Preprocessing}

Pre-processing mainly consists of two steps: skull-stripping and tensor estimation. Skull-striping is accomplished using the automatic algorithm that averages all Diffusion Weighted Images (DWI), thus creating an image of almost the same value everywhere. We use an uniformity criteria to determine what's in the brain and what is not. Tensor estimation is done in the log-euclidean (LE) space. this is how, we avoid the DWI shifting that exists in the presence of Rician noise, which commonly affect DTI datasets with low SNR. Consequently, diffusion is not underestimated as in the algebraic strategy. ${ }^{12}$ The tensor estimation that we use also includes a regularization term that keeps the edges of the tensor field while smoothing homogeneous regions. ${ }^{12}$ Preprocessing was performed in MedInria. ${ }^{13}$

\subsection{Template generation}

Once the tensors are estimated, all our datasets follow the process depicted in figure 1

The linear registration accounts for image first order differences among all our data sets, including: displacement, rotation, scaling and shearing ${ }^{14}$. Since our information comes from clinical studies that originally targeted different purposes, there were small differences in the imaging specifications. This linear registration process puts all the images in a common dimensional frame and also corrects small misalignments. For the best 


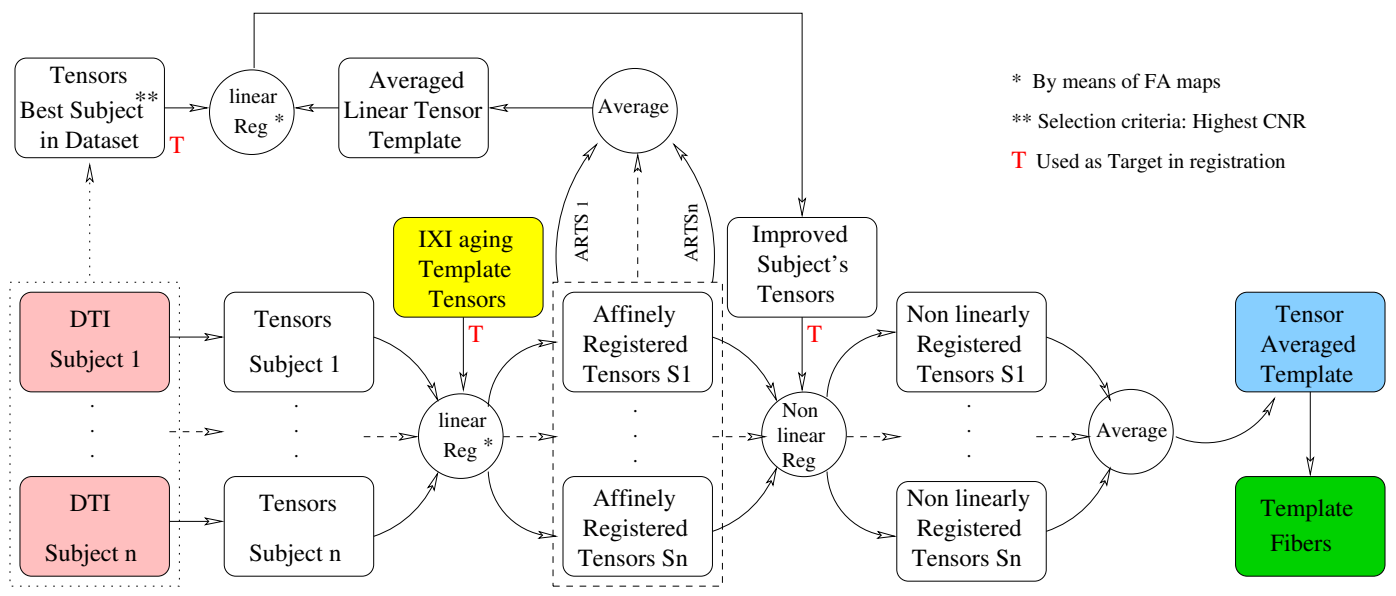

Figure 1: Schematic description of template generation

of our knowledge, there is not a defined procedure to register tensors. Consequently, we opted to do tensor registration using the transformation matrix (TM) that results from registering the FA maps of our datasets to the FA map of the IXI aging template. ${ }^{15}$ Then, the TM is applied to move the individual tensors to the tensor's space of the aging template. This procedure is fair and does not affect the integrity of the data since the linear registration only interpolates the structure of the moving samples to the fixed sample. ${ }^{14}$ Moreover, this is an intermediate step, as it would be shown later the desired tracts in each subject are obtained from the original datasets. Note that the registration procedure explained here, is used in all registering stages of this procedure.

We then average all the affinely registered tensors to create a temporal template. This volume is compounded of blurry images. In order to retain details in the template, it follows a new linear registration, in which the fixed data is the temporal template and the moving data is the best acquisition subject among our datasets. The best acquisition was selected in terms of contrast-to-noise ratio. Through linear registration, a improved subject which carries averaged information of 6 original subjects as well as details of the best subject in datasets is created.

With the aim of correcting local deformations, non-linear registration is required in inter-subjects studies. In our pipeline, non-linear registration algorithm described in $^{16}$ is used. In this step, all the affinely registered individuals are non-linearly registered into the improved subjects, as mentions above. The non-linearly registered tensors are averaged in order to create the final template, which is the local gold standard for all the experiments used in its creation. From this template we obtain the tensors and the tracts. Tensor registration is implemented using MedInria. ${ }^{13}$

\subsection{Subject warping}

Subject warping is depicted in figure 2

In order to obtain the tensors field and the tractography in each subject of our dataset, the subject's tensors must be linearly registered to the tensors of the local template. Then, a non-linear process is run in order to create the deformation fields, which are force vectors lying in each voxel. The direction and strength of the deformation fields are dependent of the differences between the FA maps of the subject and the template. ${ }^{17}$ Finally, the deformation fields are applied to the tracts in the template, creating the tracts in the subject. This warping step is executed using MedInria. ${ }^{13}$

\subsection{Neonatal brain development marker tracts}

We proceed to test our template-based method by obtaining the brain development marker tracts mentioned in section 1. For this purpose, we chose two regions of interest (ROI) and applied an AND operator in the fiber 


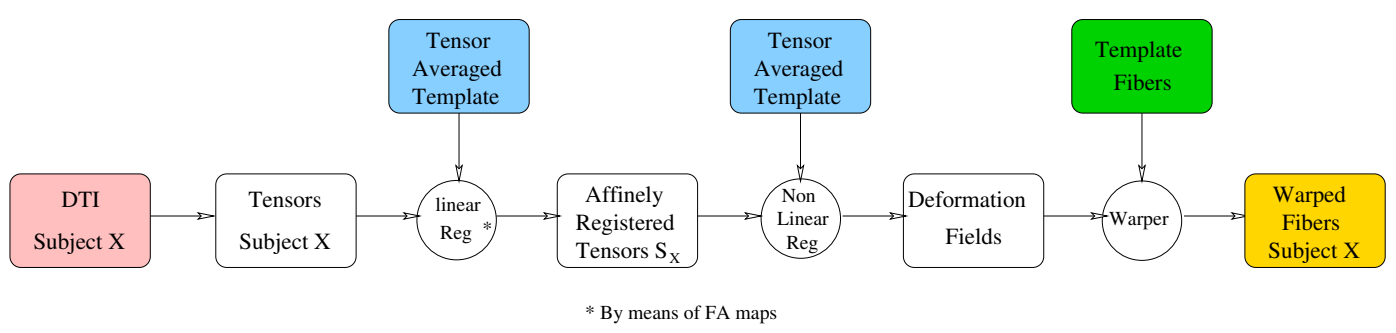

Figure 2: Warping strategy for subject tractography

tracking algorithm. These procedure was done using MedInria. ${ }^{13}$ ROI positioning in the template is determined by an expert pediatric neuroradiologist. For the fornix, one ROI is located in the body of the fornix and the other in the columns zone. For the IFOF, a coded-colored FA map is used. The first ROI is located in the green colored path that connects the temporal and parietal lobes in an image that appears few slices anterior of the splenium of the corpus callosum in a coronal view. Second ROI was located in an slice anterior to the fornix, in a circular green spot that traverses the coticospinal tract. ${ }^{18}$

For the ILF we proceed similarly to the IFOF. In the FA coded-colored map we identified the coronal slice that crosses the posterior edge of the cingulum. Then, we selected as first ROI all colored zones included in the hemisphere of interest. Second ROI was placed in the most posterior coronal slice in which the frontal lobe is disconnected from the temporal lobe. Then all fibers in the temporal lobe were selected. ${ }^{18}$

For the ATR, first ROI was placed in the entire thalamus on the coronal image, and the second one was placed in the anterior limb of the internal capsule. ${ }^{18}$ For the posterior thalamic radiations (PTR), first ROI was drawn in the entire thalamus on a coronal image and a the second one was placed in the occipital lobe. ${ }^{18}$

FA levels of 0.1 and 0.05 were chosen as trigger and stopping conditions for all tracts presented in this manuscript. 


\section{RESULTS}

\subsection{Limbic Structure: The fornix}

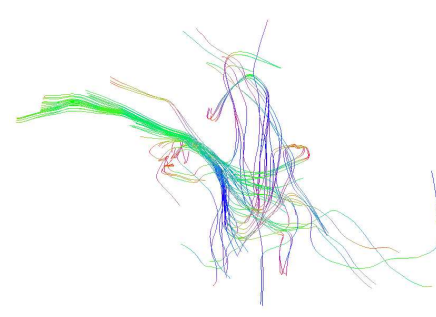

L

(a) Fornix. Subject

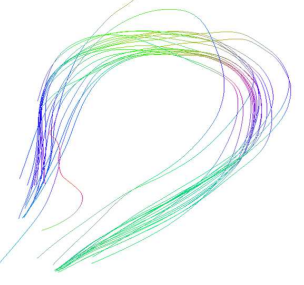

L

(d) Fornix. Template

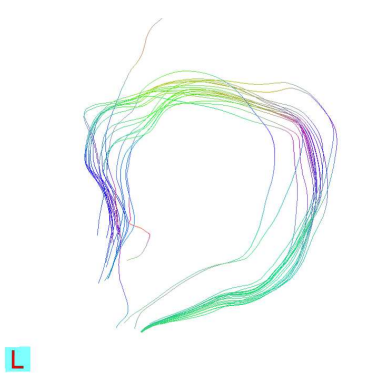

(g) Fornix. Warped subject

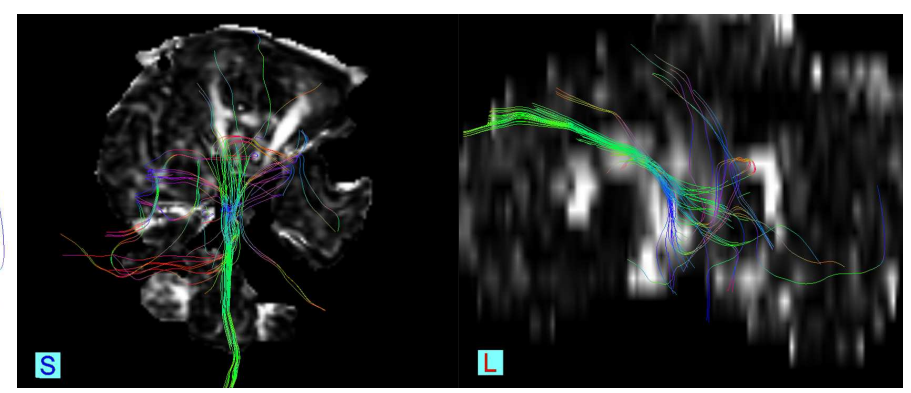

(b) Subject. Axial view

(c) Subject. Sagittal view

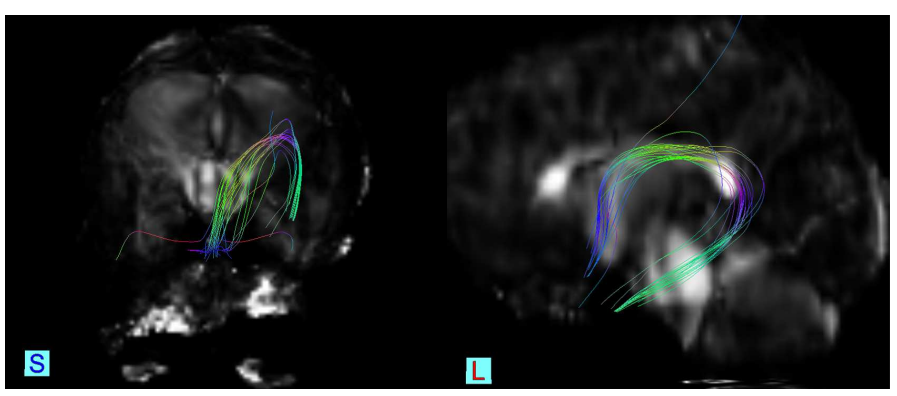

(e) Template. Axial view

(f) Template. Sagittal view

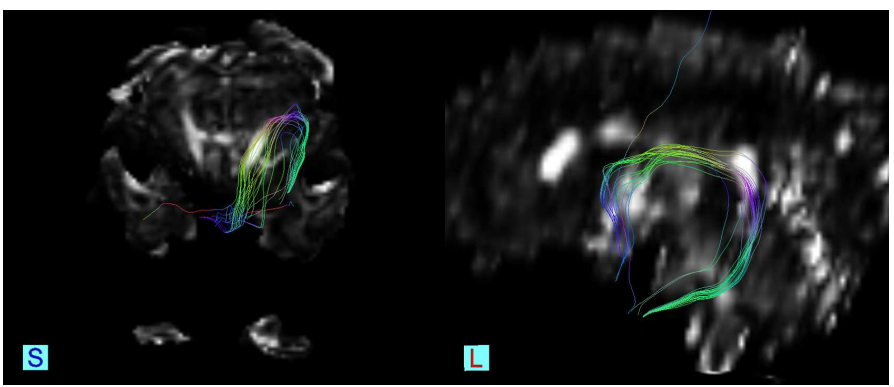

(h) Warped Subject. Axial view (i) Warped subject. Sagittal view

Figure 3: Fornix tract. Rows(from top to bottom): in a subject, in the template and in the warped subject. Columns (from left to right): stand alone tracts, tracts in axial view and tracts in sagittal view.

The fornix is located near the splenium of the corpus callosum and diverges from the mid sagittal zone at the level of the crus just before the fibers reach the hippocampus. ${ }^{19}$ The correct location of the fornix in our findings, just below the corpus callosum is well appreciated in the sagittal views of both, the template and the warped subjects. The extracted fornix presents red colored fibers at the level of the crus which is consistent with the lateral displacement anatomically described in the literature. 


\subsection{Association structures: The IFOF and the ILF}

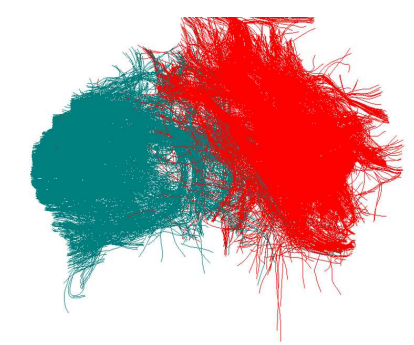

L

(a) IFOF and IFL. Subject

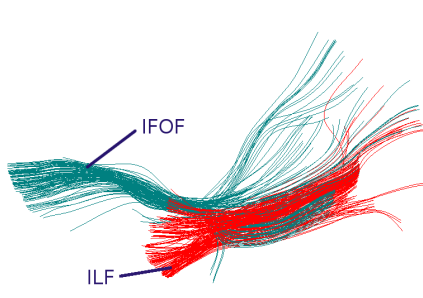

L

(d) IFOF and IFL. Template

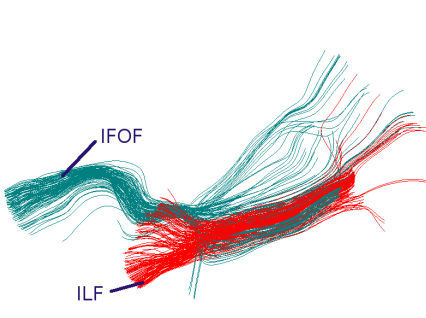

L

(g) IFOF and IFL. Warped subject

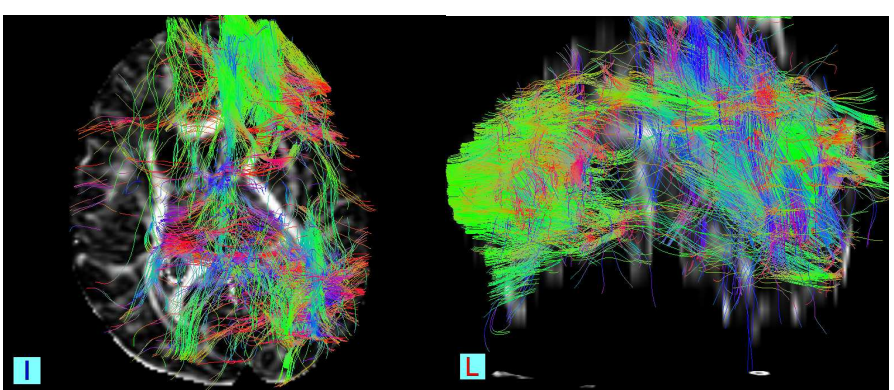

(b) Subject. Axial view

(c) Subject. Sagital view

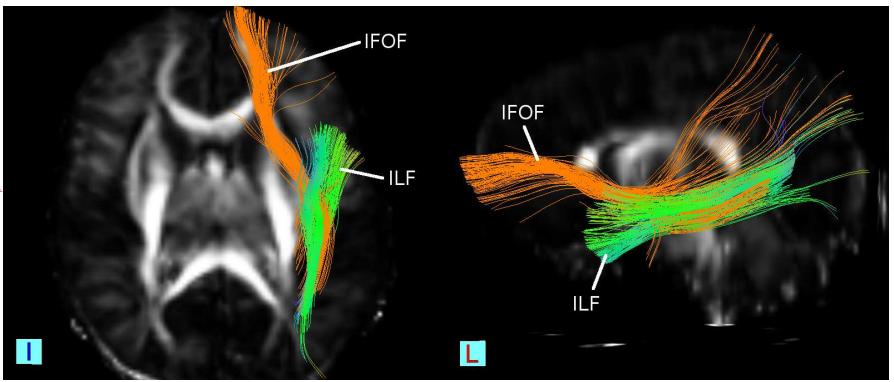

(e) Template. Axial view

(f) Template. Sagittal view

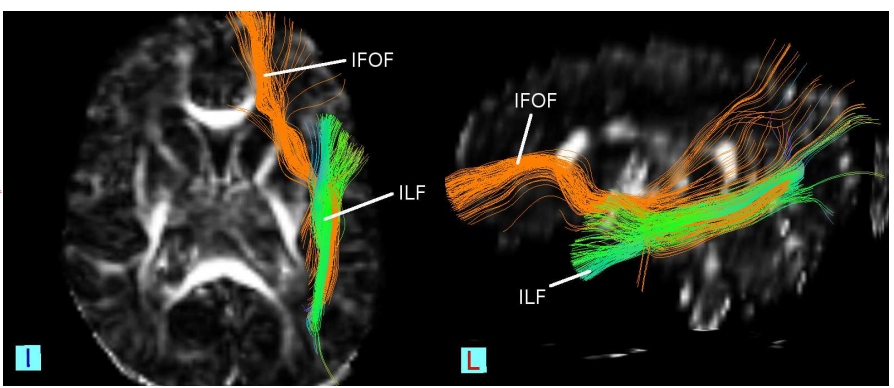

(h) Warped Subject. Axial view

(i) Warped subject. Sagittal view

Figure 4: Association tracts (IFOF and ILF). Rows(from top to bottom): in a subject, in the template and in the warped subject. Columns (from left to right): stand alone tracts, tracts in axial view and tracts in sagittal view.

Directional color-coding in the tracts was not used in order to avoid confusion in the visualization of the tracts.

The ILF connects the occipital cortex with the anterior temporal lobe and amygdala, whereas the IFOF begins in the occipital cortex, continues medially through the temporal cortex dorsal to the uncinate fasciculus, terminating in the orbitofrontal cortex. ${ }^{20}$ Figures $4 \mathrm{~d}-4 \mathrm{i}$, are consistent with this description. 


\subsection{Commisural structures: The ATR and the PTR}

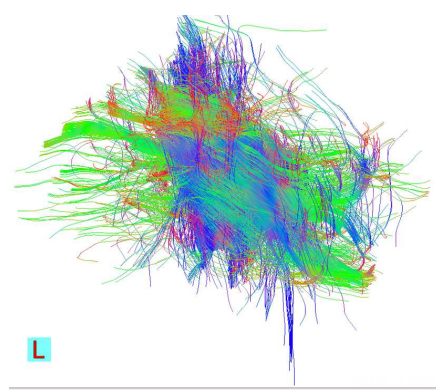

(a) ATR and PTR. Subject

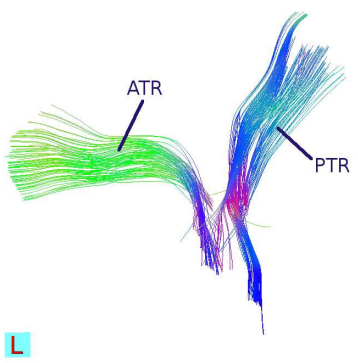

(d) ATR and PTR. Template

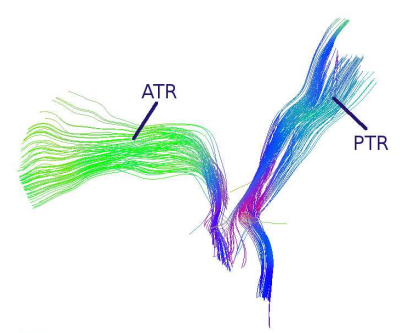

L

(g) ATR and PTR. Warped subject

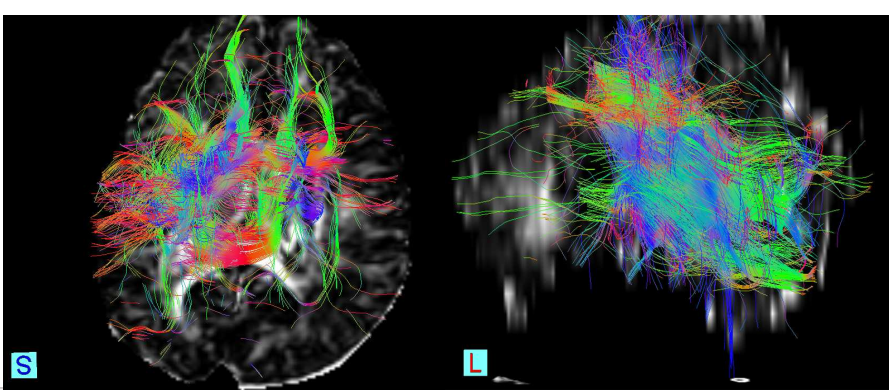

(b) Subject. Axial view

(c) Subject. Sagital view

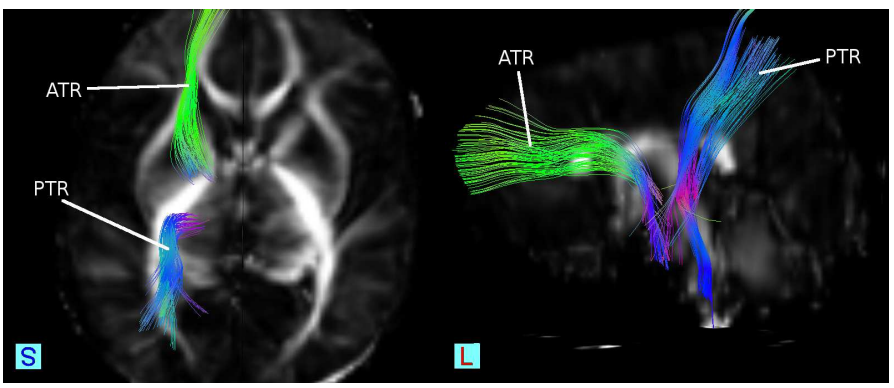

(e) Template. Axial view

(f) Template. Sagittal view

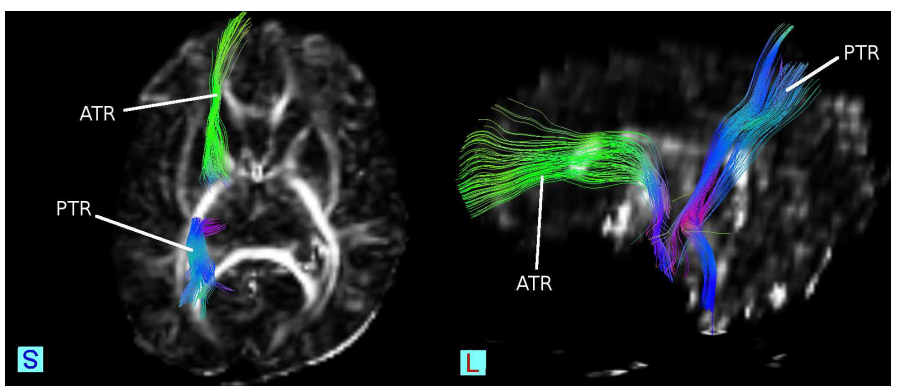

(h) Warped Subject. Axial view

(i) Warped subject. Sagittal view

Figure 5: Commisural tracts. Rows(from top to bottom): in a subject, in the template and in the warped subject. Columns (from left to right): stand alone tracts, tracts in axial view and tracts in sagittal view.

The ATR consists of fibers between mediodorsal thalamic nuclei and the frontal cortex, and fibers between anterior thalamic nuclei and the anterior cingulate cortices. ${ }^{21}$ This is better seen in Figure 5i, where the contrast of the overlapped FA map permits the localization of the tract in the brain.

\section{DISCUSSION}

In this manuscript we present a method that recovers meaningful information from tractography in datasets that originally do not have the required quality to do so. The efficacy of the pipeline was proven by obtaining tracts usually difficult to see when direct tractography is used. The accuracy of the method lies in the fact that the created local template ends by having much more quality than any of its contributors. ${ }^{22}$ The averaging used to create the template ideally improves in $\sqrt{n}$ times the SNR. But this is not the only one improving strategy in our methods. In addition, the registering processes depicted in figures 1 and 2 are executed in the tensors field, and 
include a tensor rotation operation before averaging. With the tensor rotation we assure the correct structural overlapping trough subjects so the important information is not taken as non-correlated data in the averaging process. This procedure gains more relevance when considering the usual lack of uniformity of the clinical data.

According to Prayer et al, ${ }^{10}$ cerebral myelination is a predominantly postnatal process, progressing in a craniocaudal direction and centrifugal manner. Figure 5h shows shorter PTR projections to the occipital lobe, respect to the same view in the template. See figure 5e. This suggest a growing pattern in accordance to the anatomical known dynamics mentioned above.

Other remarkable fact of our procedure is that all the resources are obtaining from the data itself, meaning that not external resources are needed, except of the aging template that is publicly available. This fact gives a high degree of repeatability. Scalability is also granted, if the same procedure is applied in data with better quality the improvement will be still present.

\section{CONCLUSIONS}

A method to process information from archived-low quality-DTI datasets has been proposed and tested. It uses no other images than the same datasets to be treated and the IXI Aging DTI atlas that is publicly available.

Our procedure is suitable for clinical DTI data which is abundant in hospitals but often not usable for research due to its lack of uniformity. Hospitals keep this data for legal reasons but it is usually stored with no prospect for further use. Recovering this information is of outstanding importance since it represents one of the most extensive database covering controls, pathologies, and special cases. Our method makes from the stored DTI medical images, a constant growing source of material for rich background referencing, actual procedure improvement and research.

We have also demonstrated that our warping strategy is loyal to the FA information in the subjects even when more complete tracts are drawn in the template. This is a remarkable characteristic of the method and, since the development of marker tracts is a dynamic process, the proposed method becomes a promising tool to construct atlases of baby brains that can be accomplished grouping by age and developmental level.

This method could be extended to the study of those embryonic-associated illnesses that are nowadays studied based in the analysis of sequela. ${ }^{23,24}$ As we made hardly extractable brain structures such as the ones presented in this manuscript to be visible under tractography methods, the studies of embryonic/fetus brain associated illnesses can migrate to the temporal origin of the clinical complication.

\section{REFERENCES}

[1] Huppi, P. S. and Dubois, J., "Diffusion tensor imaging of brain development," Seminars in Fetal ES Neonatal Medicine 11, 489-497 (2006).

[2] Huang, H., Zhang, J., Wakana, S., Zhang, W., Ren, T., Richards, L. J., Yarowsky, P., Donohue, P., Graham, E., van Zijl, P. C. M., and Mori, S., "White and gray matter development in human fetal, newborn and pediatric brains," NeuroImage 33, 27 - 38 (2006).

[3] Cascio, C. J., Gerig, G., and Piven, J., "Diffusion Tensor Imaging: Application to the Study of the Developing Brain," J. AM. ACAD. CHILD ADOLESC. PSYCHIATRY 46, 213-223 (2007).

[4] Basser, P. J., Mattiello, J., and LeBihan, D., "MR diffusion tensor spectroscopy and imaging," Biophys. J. 66(1), $259-267$ (1994).

[5] Jonathan Guillard, A. W. and Barker, P., [Clinical Neuroimaging], ch. 5, Cambridge University Press (2005).

[6] Liu, T., Li, H., Wong, K., Tarokh, A., Guo, L., and Wong, S. T. C., "Brain tissue segmentation based on DTI data," Neuroimage 38, 114 - 123 (2007).

[7] Elysia, A. M., Vann, C. M. D., Kenneth, J. P. M., Ruth, E. G. P., Anne, S. M., and Mas, M. S. P. M., "Tractography-Based Quantitation of Corticospinal Tract Development in Premature Newborns," The Journal of Pediatrics 156(6), $882-888$ (2009).

[8] Walhovd, K., Westlye, L., Moe, V., Slinning, K., Due-Tnnessen, P., Bjornerud, A., van der Kouwe, A., Dale, A., and Fjell, A., "White matter characteristics and cognition in prenatally opiate- and polysubstanceexposed children: A diffusion tensor imaging study," American Journal of Neuroradiology 31(5), 894-900 (2010). 
[9] Liu, Y., Balériaux, D., Kavec, M., Metens, T., Absil, J., Denolin, V., Pardou, A., Avni, F., Bogaert, P. V., and Aeby, A., "Structural asymmetries in motor and language networks in a population of healthy preterm neonates at term equivalent age: A diffusion tensor imaging and probabilistic tractography study," NeuroImage 51(2), 783 - 788 (2010).

[10] Prayer, D., Kasprian, G., Krampl, E., Ulm, B., Witzani, L., Prayer, L., and Brugger, P. C., "Mri of normal fetal brain development," European Journal of Radiology 57(2), 199 - 216 (2006).

[11] Dubois, J., Hertz-Pannier, L., Dehaene-Lambertz, G., Cointepas, Y., and Bihan, D. L., "Assessment of the early organization and maturation of infants' cerebral white matter fiber bundles: A feasibility study using quantitative diffusion tensor imaging and tractography," NeuroImage 30, 1121 - 1132 (2006).

[12] Fillard, P., Pennec, X., Arsigny, V., and Ayache, N., "Clinical DT-MRI Estimation, Smoothing and Fiber Tracking with Log-Euclidean Metrics," IEEE Transactions on Medical Imaging 26(11), 1472 - 1482 (2007).

[13] Toussaint, N. et al., "Medinria: Medical image navigation and research tool by inria," in [Workshop on Interaction in medical image analysis and visualization], MICCAI'07 (2007).

[14] Jenkinson, M. and al. et, "Improved Optimization for the Robust and Accurate Linear Registration and Motion Correction of Brain Images," NeuroImage 17, 825 - 841 (2002).

[15] Zhang, H. et al., "A tract-specific framework for white matter morphometry combining macroscopic and microscopic tract features," Medical Image Analysis 14(5), 666-673 (2010).

[16] Yeo, B. T. T., Vercauteren, T., Fillard, P., Peyrat, J.-M., Pennec, X., Golland, P., Ayache, N., and Clatz, O., "DT-REFinD: Diffusion Tensor Registration with Exact Finite-Strain Differential," IEEE Transactions on Medical Imaging 26(12), 1472 - 1482 (2009).

[17] Thye, B. et al., "Dt-refind: Diffusion tensor registration with exact finite-strain differential.," in [IEEE Transactions on Medical Imaging], 28(12), 1914-1928 (2009).

[18] Wakana, S. et al., "Reproducibility of quantitative tractography methods applied to cerebral white matter," Neuroimage 3, 630-44 (Jul 2007).

[19] Kendi, M., Kendi, A. T. K., Lehericy, S., Ducros, M., Lim, K. O., Ugurbil, K., Schulz, S. C., and White, T., "Structural and diffusion tensor imaging of the fornix in childhood and adolescent onset schizophrenia," Journal of the American Academy of Child and Adolescent Psychiatry 47(7), 826 - 832 (2008).

[20] Philippi, C. L., Mehta, S., Grabowski, T., Adolphs, R., and Rudrauf, D., "Damage to association fiber tracts impairs recognition of the facial expression of emotion," The Journal of Neuroscience 29(48), 15089-15099 (2009).

[21] Mamah, D., Conturo, T. E., Harms, M. P., Akbudak, E., Wang, L., McMichael, A. R., Gado, M. H., Barch, D. M., and Csernansky, J. G., "Anterior thalamic radiation integrity in schizophrenia: A diffusion-tensor imaging study," Psychiatry Research: Neuroimaging 183(2), 144 - 150 (2010).

[22] Goodlett, C. B. et al., "Group analysis of dti fiber tract statistics with application to neurodevelopment," in [Neuroimage], 45, 133-42 (2009).

[23] Eikenes, L., Løhaugen, G. C., Brubakk, A.-M., Skranes, J., and Håberg, A. K., "Young adults born preterm with very low birth weight demonstrate widespr ead white matter alterations on brain DTI," NeuroImage 54, 1774 - 1785 (1 February 2011).

[24] Mullen, K. M., Vohr, B. R., Katz, K. H., Schneider, K. C., Lacadie, C., Hampson, M., Makuch, R. W., Reiss, A. L., Constable, R. T., and Ment, L. R., "Preterm birth results in alterations in neural connectivity at age 16 years," NeuroImage 54, 2563 - 2570 (14 February 2011). 